\title{
Nanoengineered Colloidal Probes for Raman-based Detection of Biomolecules inside Living Cells
}

\author{
Alexey Yashchenok, Admir Masic, * Dmitry Gorin, Bong Sup Shim, \\ Nicholas A. Kotov, Peter Fratzl, Helmuth Möhwald, and Andre Skirtach*
}

Nanosized tools for interrogating and handling cells are becoming important means in obtaining information about cell functions. Glass micropipettes and capillaries, ${ }^{[1]}$ carbon nanopipettes and endoscopes ${ }^{[2]}$ are some of the devices which have been recently developed. However, inserting even these objects through the cell membrane leaves the membrane open during investigation prompting for development of a probe which can be incorporated intracellularly for remote detection and enhanced imaging of biomolecules. Typically, information about molecules in cellular environments is obtained by confocal microscopy which is a noninvasive but which uses fluorescent labels and markers. ${ }^{[3]}$ In this regard, confocal Raman spectroscopic imaging is becoming increasingly popular for label-free imaging of cells, due to its nature of scattering inherent to all biomolecules ${ }^{[4]}$ (Figure S1 in Supporting Information). However, its applicability is hindered by the weakness of the detected signals and high intensity of laser which is required to obtain reliable information (potentially introducing the problem of beam damage and hindering living cells studies). ${ }^{[5]}$ One solution to this problem could be amplification through surface enhancement, SERS, ${ }^{[6-9]}$ which provides powerful means of signal amplification. It has been previously employed for molecular detection with high sensitivity recognition of an analyte, ${ }^{[10]}$ as well as for single molecule detection at $10^{8}$ amplification. ${ }^{[11,12]}$ Such an enhancement of the Raman signal is due to excitation of the surface plasmons and/or the formation of a charge-transfer state at the surface of metal nanoparticles, ${ }^{[13]}$ in this way significant response of SERS can be achieved in hotspots located between nanoparticles. ${ }^{[14-17]}$ It was previously observed that nanoshells and dimers ${ }^{[18,19]}$ as well as nanorods ${ }^{[20]}$ can produce a strong enhancement of individual nanoparticles Another interesting application of SERS is to act as an intracellular sensor, for example, for $\mathrm{pH} .^{[21]}$

It was previously reported that gold nanoparticles (AuNPs) attached to the surface of carbon nanotubes increase Raman scattering of the G-band of carbon nanotubes. ${ }^{[22,23]}$ Increasing the size of nanoparticles and decreasing the distance between them and carbon nanotubes led to dramatically increased Raman signals. ${ }^{[24,25]}$ On the other hand, intracellular insertion of probes, mainly particles and capsules ranging from nanometer to micrometer scale, was widely applied by several groups. ${ }^{[26-29]}$ Incorporation such carriers has potentiality for analysis of biomolecules, ${ }^{[27]}$ and drug delivery inside living cells ${ }^{[30,31]}$ as well as interaction of molecules relevant for many areas of cell biology. ${ }^{[32]}$

In this work we engineered colloidal probes by functionalizing their surface with single-wall carbon nanotubes (SWCNT) and AuNP aggregates. Subsequently, we incorporated them into the cytosol of NIH3T3 fibroblast cells using a new method based on electroporation ${ }^{[33]}$ and exploited intracellular SERS to study biomolecules in both living and fixed
Dr. A. Yashchenok, Prof. D. Gorin, Prof. H. Möhwald, Prof. A. Skirtach

Max Planck Institute of Colloids and Interfaces

Department of Interfaces

14424 Potsdam, Germany

E-mail: andre.skirtach@ugent.be

Dr. A. Masic, Prof. P. Fratzl

Max-Planck Institute of Colloids and Interfaces

Department of Biomaterials

14424 Potsdam, Germany

E-mail:masic@mpikg.mpg.de

Dr. A. Yashchenok, Prof. D. Gorin

Saratov State University

Department of Nano- and Biomedical Technologies

410012 Saratov, Russia
Prof. D. Gorin

Saratov State University

Institute of Nanostructures and Biosystems

410012 Saratov, Russia

B. S. Shim, Prof. N. A. Kotov

University of Michigan

Department of Chemical Engineering

Department of Materials Science

Department of Biomedical Engineering

Biointerface Institute,

Ann Arbor, MI 48109, USA

Prof. A. Skirtach

Department of Molecular Biotechnology

Ghent University

9000 Ghent, and Nano-Bio, (NB)-Photonics

Ghent University

9000 Ghent, Belgium

DOI: 10.1002/smll.201201494 


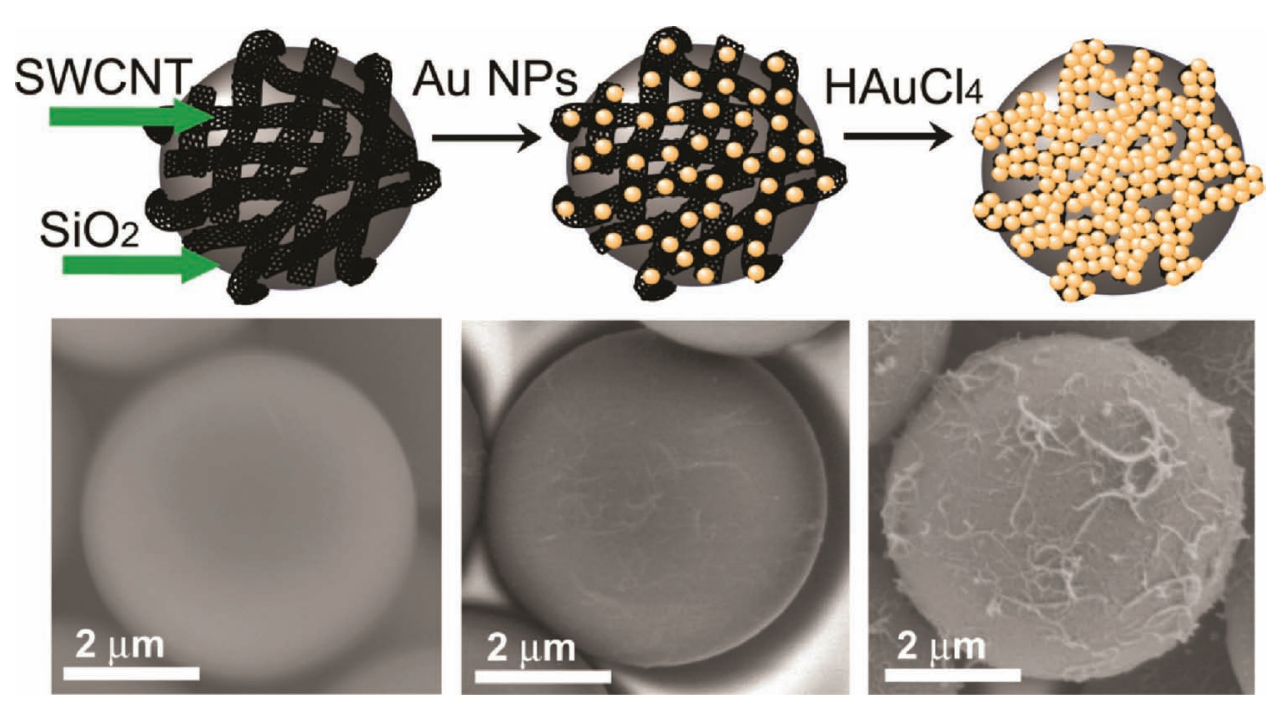

Figure 1. Schematics (top) and environmental scanning electron microscope (ESEM, bottom) images of colloidal probes developed in this study. Probes were prepared using the layer by layer (LbL) method by adsorption of SWCNT (left panel), deposition of AuNP (central panel) and subsequent chemical growth of gold nanoparticles (right panel).

cells. Such an intracellular incorporation methodology can be applied to any cell type, and it has already been successfully used for studying intracellular trafficking and the surface presentation of small peptides-MHC 1 class complex. ${ }^{[33]}$

Figure 1 shows the schematics of probe functionalization and their respective environmental scanning electron microscopy (ESEM) images. Silica particles are very smooth, while adsorption of SWCNT (Figure 1, left panel) increases the roughness of their surface. The latter further increases upon adsorption of AuNP (Figure 1, middle panel). It can be noted that direct adsorption of nanoparticles at high concentrations or reduction of metal salts can lead to formation of aggregates: ${ }^{[34]}$ a feature that contributes to the Raman signal enhancement. In this work we combine both of these features, first, by adsorbing nanoparticles at high concentration, followed by chemical growth of gold nanoparticles (or in other words reduction of gold salts) to produce highly efficient SERS probes (Figure 1, right panel). Throughout this work we refer to aggregates as to those which were obtained by latter procedure.

The Raman spectroscopic signature of SWCNT allows sensing the magnitude of SERS amplification (Figure 2a), which is significant for localization of the probes. It can be seen from Figure 2a that the intensity of the characteristic G-band is increased at least by a factor of 60 for the probes with AuNPs aggregates (Figure 2a, red curve) compared to that for the untreated sample (Figure 2a, black curve). The inset in Figure 2 shows an optical (top) and a Raman (bottom) image acquired with confocal Raman microscopy. Raman imaging (red color in inset) was obtained by integrating the G-band of SWCNT $\left(1530-1630 \mathrm{~cm}^{-1}\right)$. Probes treated with AuNP aggregates appear black in the transmission image and show high and, notably, constant enhancement of the Raman signal, whereas those without nanoparticles appear grey in the transmission image and are characterized by a weak Raman signal. It is clear that SWCNTs provide increased roughness for deposition of AuNP ${ }^{[35]}$ essentially contributing to the enhancement. To monitor gold deposition at all steps we deposited the same compositions (SWCNT, SWCNT+AuNP, and SWCNT+AuNP+reduction) on planar

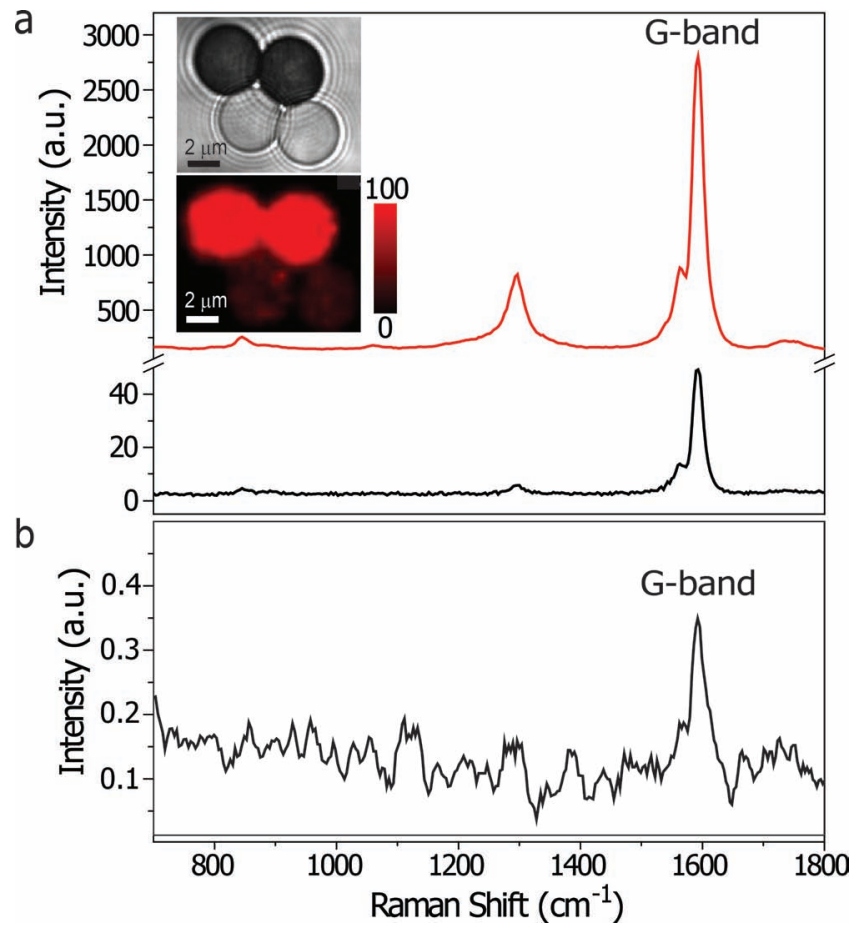

Figure 2. Raman spectra obtained from colloidal probes covered with SWCNT and gold nanoparticle aggregates. a) Raman spectra from the surface of the probes covered with SWCNT (black line) and probes covered with SWCNT subsequently covered with gold nanoparticle aggregates (red line). Inset shows Confocal Laser Scanning Microscope transmission (top) and Raman (bottom) reconstructed images; red contrast in the Raman image was produced by integration of the G-band. All spectra were acquired with a laser operating at $0.1 \mathrm{~mW}$. b) Raman spectrum of probes functionalized with SWCNT covered with gold nanoparticle aggregates obtained with a laser operating at $0.1 \mu \mathrm{W}$. All spectra were acquired using a laser with $785 \mathrm{~nm}$ wavelength. 

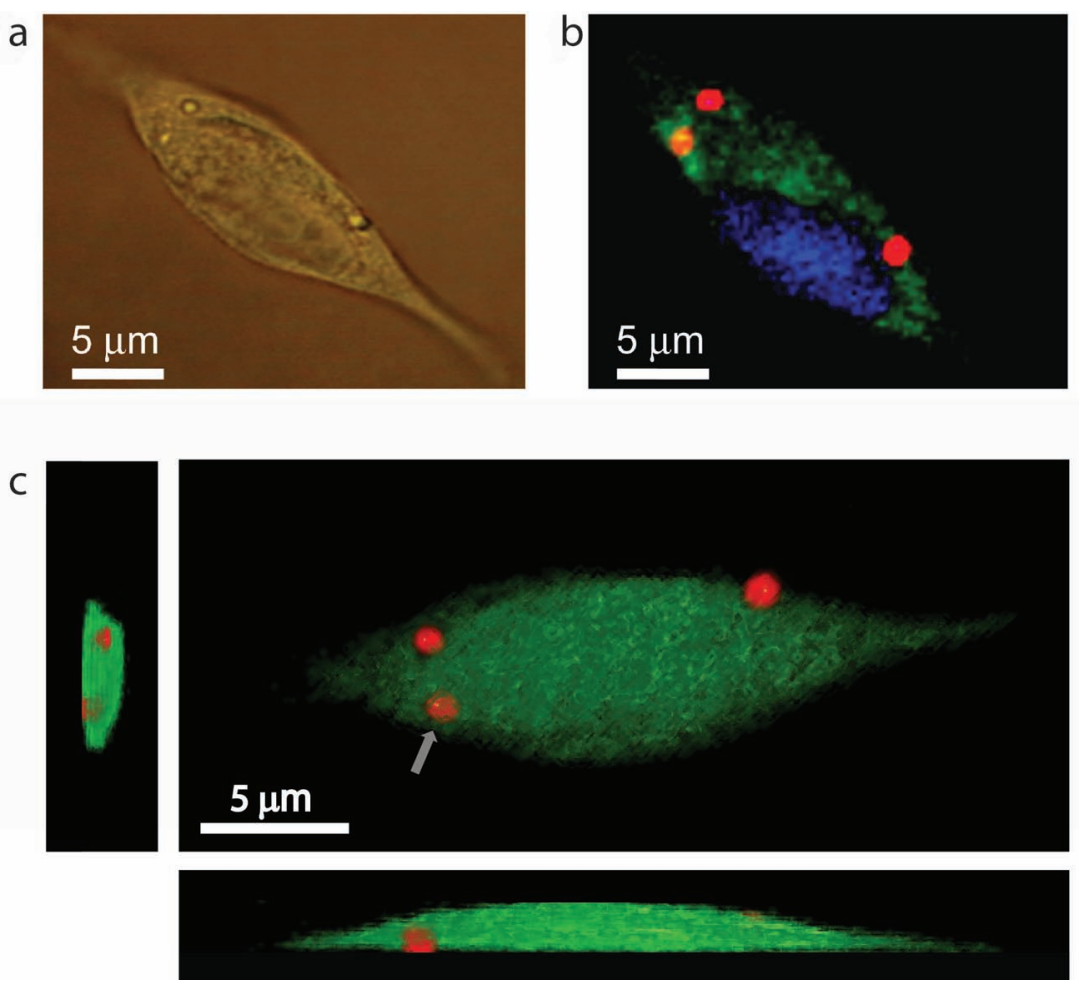

Figure 3. Raman spectroscopic imaging of a fixed fibroblast cell with electroporated colloidal probes. a) Optical image of the analyzed cell. b) Confocal Raman image of the same cell in (a) acquired at a specific depth. Color codes refer to differences in Raman spectral features in the data set (green-cytosol, blue-nucleus, and red-SWCNT). (c) 3D reconstruction of data set where green represents cell organic content ( $\mathrm{C}-\mathrm{H}$ stretching) and red SWCNT (G-band) distribution. Raman images were acquired using integration time of $0.1 \mathrm{~s}$ and laser operating at $532 \mathrm{~nm}$.

films (Figure S2 in Supporting Information). These data illustrate the substantial increase of absorption after chemical growth of AuNP and also reveal the surface plasmon peak broadening (Figure S2 in Supporting Information). Nanoengineered probes allow for observation and enhancement of the G-band of SWCNT at extremely low laser powers $(\sim 0.1 \mu \mathrm{W}$ at the sample, Figure $2 b$ ) that can be compared to the power of sun light. Low laser power in combination with the characteristic and reliable SWCNT Raman spectral signal shows the potential for real-time localization and identification of probes in living systems and tissues. In the next steps we used these probes for studying fixed and living cells.

Fixing cells is a standard procedure to visualize various cellular sub-compartments upon labeling. Figure 3 shows optical micrograph and Raman confocal microscopy image of a fixed cell with incorporated colloidal probes. The optical image (Figure 3a) allows identification of probes, whereas the Raman imaging clearly shows three particles distributed in different regions of the cell (Figure 3b). Furthermore, analysis of Raman spectral features allows differentiation between nucleus (blue) and cytosol (green) without the need of any molecular labeling (compare with Figure S1 in Supporting Information). The versatility of Raman confocal imaging is evident in Figure 3c where a 3D reconstruction of Raman features are shown (see also Video in Supporting Information). It is clear that one colloidal probe pointed at by the grey arrow is located inside the cell. For comparison, the confocal laser scanning fluorescence microscope (CLSM) image of a similar cell, in which the cytosol and nucleus are stained with TRITCphalloidin and TO-PRO-iodide, is shown in Figure S3, Supporting Information. Figure S4 shows cells with incorporated probes. Although different organelles can be mapped by CLSM and Raman imaging, in the latter case information is gathered by analyzing molecular spectral features without labels. ${ }^{[36,37]}$

Figure 4a presents the Raman imaging of a living cell with incorporated AuNP aggregates-SWCNT functionalized silica probes. Color contrast for cytosol and nucleus was obtained from the typically weak non-resonance Raman signals shown in Figure 4b (blue-nucleus, green-cytosol, yellow-carotenoids). Here, used laser power of $0.1 \mathrm{~mW}$ (wavelength $532 \mathrm{~nm}$ ) does not allow for reliable Raman sensing of biomolecules. The solution for this problem could be an increase of laser power (Figure S1 for a fixed cell in Supporting Information) or decrease of laser wavelength. The increase of laser power was shown to affect living cells, while "bio-friendly" near-infrared lasers ${ }^{[38]}$ operates at even higher wavelengths, thus further reducing Raman scattering intensity. Another interesting feature of the proposed approach is the presence of a particularly intense signal from SWCNT (Figure 4b, red curve), which allows for facile tracking of the probes in the cell, upon intracellular incorporation. In addition, very strong SERS signals were acquired from the surface of the probe (Figure 4c); two different Raman spectra were recorded from two different regions. The features in the SERS spectra can be attributed to characteristic peaks of biomolecules in living cells. ${ }^{[4,39]}$ Specifically, probes localized in the cytosol were characterized by SERS signals of proteins and lipids (Figure 4c, upper spectrum). Also in addition to protein bands, the probe reveals peaks related to aromatic ring vibrations of nucleic acids of DNA/ RNA macromolecules (lower spectrum in Figure 4c, bands at $1314 \mathrm{~cm}^{-1}, 1376 \mathrm{~cm}^{-1}$ and $\left.1479 \mathrm{~cm}^{-1}\right) \cdot{ }^{[40]}$ It can be emphasized that introduction of our probes allowed for drastic increase of the signal to noise (SNR) ratio which was $\sim 5$ for a nonresonant Raman (Figure $4 \mathrm{~b}$, blue and green traces), but which became $\sim 100$ for the SERS signals. Such an increase of SNR allows for reduction of both required laser power levels and necessary spectrum integration times alleviating potential laser induced damage and enabling fast acquisition.

The possibility of acquiring label-free Raman signal ${ }^{[37]}$ of an intracellular environment and its SERS enhancement through colloidal probes is expected to open new perspectives for chemical imaging of cells. ${ }^{[33]}$ Incorporation of such probes was conducted via a recently developed electroporation 

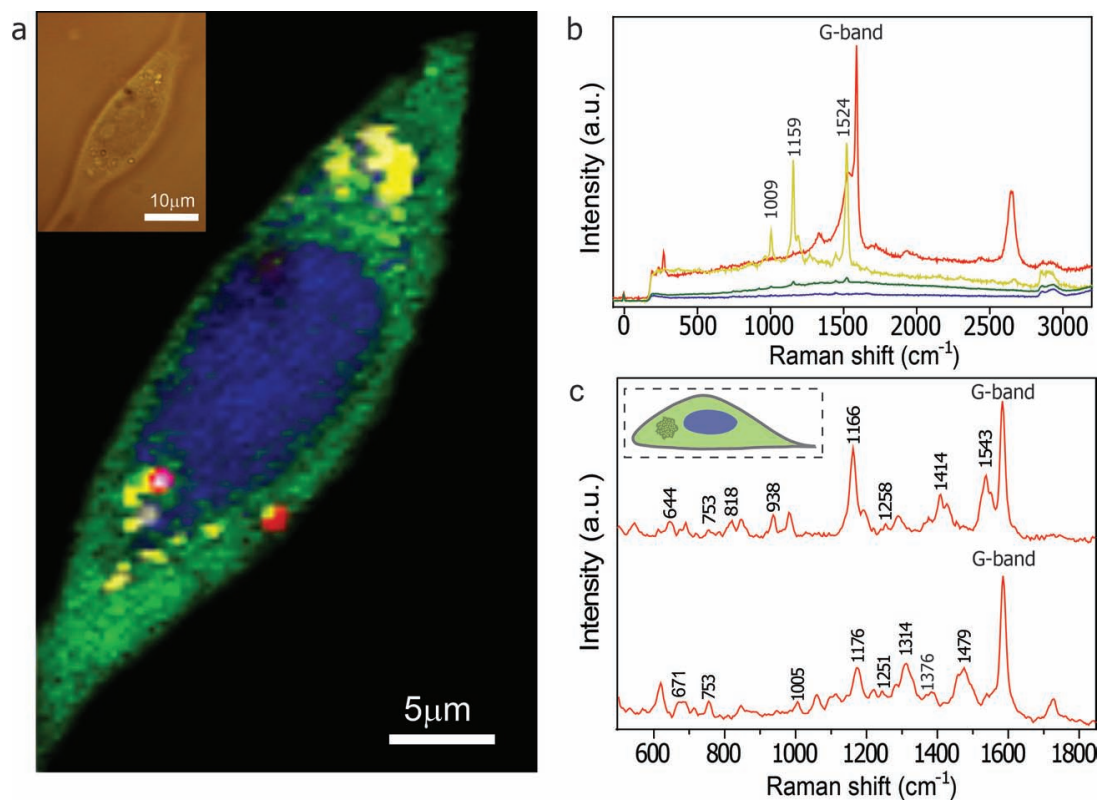

Figure 4. Raman spectroscopic imaging of a living fibroblast cell with incorporated colloidal probes. The color map in (a) reflects the differences found in the Raman data and is a linear combination of the averaged single spectra (reported in b) and characteristic for cell compartments (green-cytoplasm, blue-nucleus, yellow-carotenoids) and gold-nanoparticleSWCNT functionalized colloidal probes (red). The inset in (a) shows the optical microscope image of the same cell. (c) Intracellular SERS signal recorded at the surface of the probe. Two different data sets shown in (c) were recorded at the probe incorporated into the cell cytosol. Raman images were acquired using integration time of $0.1 \mathrm{~s}$ and laser operating at $532 \mathrm{~nm}$.

based technique. The biggest advantage of this technique is that it can be applied to any cell type, while being not disruptive to metabolism and functions of living cells. In addition, spontaneous uptake, which can be pursued with only specific cell types, ${ }^{[41-43]}$ can be used as an alternative route of intracellular incorporation. Cell viability studies upon laser illumination were conducted by observing cell division in the case of electroporation based uptake and by cell-viability test in the case of spontaneous uptake. ${ }^{[31]}$

Materials used in our work, silica particle based probes, are widely considered to be inert or in other words nonreactive in a biological environment. Such a property makes them the material of choice for coating over, for example, quatum dots (QDs) for improving biocompatibility (for example). ${ }^{[44]}$ Also, it is not only coating of other particles by silica, but also direct test showed good biocompatibility. ${ }^{[45]}$

In conclusion, we have presented a SERS platform based on silica probes coated with SWCNT subsequently functionalized with AuNP aggregates for intracellular sensing of biomolecules. Functionalized SWCNT in our study carry dual functionality: they allow for easy localization at extremely low laser powers (even with power levels comparable to sun light) and provide increased roughness necessary for highly efficient SERS amplification. The approach described here can be applied to any smooth colloidal particle converting it into a superior SERS platform. Upon intracellular incorporation probes significantly enhance molecular fingerprints of biomolecules commonly found inside cells and enable fast acquisition rates at laser powers completely harmless to living cells. Remarkable increase of SNR due to SERS allows visualization and detection of biomolecules otherwise poorly detected by the non-resonant approach. The Raman probes developed in this work can be ubiquitously applied for molecular imaging and sensing in cells and tissues.

\section{Experimental Section}

Materials: Silica microparticles $\left(\mathrm{SiO}_{2}\right)$ water suspension, average core diameter $1.55 \mu \mathrm{m}$ and $4.78 \mu \mathrm{m}$, were purchased from Microparticles $\mathrm{GmbH}$. Poly(sodium 4-styrenesulfonate) (PSS, $70 \mathrm{kDa}$ ), Poly(dial lyldimethylammoniumchloride) (PDADMAC, 250-350 kDa), double stranded Deoxyribonucleic acid sodium salt (dsDNA) were purchased from Sigma-Aldrich. Sodium chloride $(\mathrm{NaCl})$ and Potassium carbonate $\left(\mathrm{KCO}_{3}\right)$ were purchased from Merck. L-Ascorbic acid was purchased from Fluka. 4-(dimethylamino) pyridine (DMAP)-stabilized positively charged nanoparticles (PNPs) were prepared according to previously published methods; ${ }^{[46]}$ they were positively charged and had a mean diameter of 8-10 nm. Purified HiPco single wall carbon nanotubes (CNT) were purchased from Carbon Nanotechnologies Inc. (CNI). Alexa Fluor 488 phalloidin and TRITC-phalloidin (Invitrogen, Eugene, Oregon, USA). The water used in all experiments was prepared in a three-stage Milli-Q Plus 185 purification system and had a resistivity higher than $18.2 \Omega \mathrm{cm}$.

Characterization: Environmental scanning electron microscopy (ESEM) imaging was recorded with a high-resolution low vacuum FEI Quanta 600 FEG instrument operating at $30 \mathrm{kV}$ with extended low-vacuum environment.

Core-Shell Structure Fabrication: Silica microparticles were used for producing core shell structures. The first step includes washing silica spheres in deionised water for 3 times. After the washing step layer-by-layer assembly was employed for fabrication of core shell carbon nanotube structures onto the silica surface. The shell composition comprised several layers of polyelectrolytes with embedded carbon nanotubes. PDADMAC and PSS were used as polyelectrolytes with concentration $2 \mathrm{mg} / \mathrm{mL}$ in $0.5 \mathrm{M} \mathrm{NaCl}$ water solution. Firstly PDADMAC was adsorbed onto the silica surface incubated in such a polyelectrolyte solution for 15 min followed by three times washing in deionised water. In addition PSS was terminated to the PDADMAC layer by the same procedure. Following layers of PDADMAC and PSS were deposited on the silica spheres as described above. As a result (PDADMAC/PSS) 3 /PDADMAC composition of multilayers was fabricated. Due to stabilization by PSS the CNTs have a negative charge surface and the Zeta-potential of PSS-stabilized single wall carbon nanotubes is $-64.1 \pm 0.2 \mathrm{mV}$. The initial suspension of CNTs was diluted in deionised water with a ratio $1 / 10(\mathrm{v} / \mathrm{v})$. The dispersion was continuously shaken during $15 \mathrm{~min}$ followed by three times centrifugation and washing in deionised water. The initial dispersion of positively charged gold nanoparticles (PNPs) was diluted in deionised water with a ratio 
$1 / 10(v / v)$. One millilitre of PNPs-diluted was added to the coated silica microparticles and was shaken during 15 min followed by centrifugation and washing in deionized water at least two times. As a result (PDADMAC/PSS) 3 /PDADMAC/CNTs/PNPs multilayers were fabricated on a silica core.

Reduction of Gold Salt: To chemical growth the gold nanoparticles on the (PDADMAC/PSS) 3 /PDADMAC/CNTs/PNPs silica microparticles, we used a technique as was described earlier. ${ }^{[47]} \mathrm{At}$ first $25 \mathrm{mg}$ potassium carbonate $\left(\mathrm{K}_{2} \mathrm{CO}_{3}\right)$ was dissolved in $100 \mathrm{~mL}$ of deionized water. This mixture was stirred for $10 \mathrm{~min}$, then $1.5 \mathrm{~mL}$ of a solution of $1 \% \mathrm{HAuCl}_{4}$ in water was added. After vigorous stirring for $30 \mathrm{~min}$ a $4 \mathrm{~mL}$ aliquot of the colorless solution, we injected $200 \mu \mathrm{L}$ of the solution containing the (PDADMAC/ PSS) ${ }_{3} /$ PDADMAC/CNTs/PNPs silica beads. We then added a $10 \mu \mathrm{L}$ (1\%) aliquot of L-ascorbic acid. Over the course of $30 \mathrm{~s}$, the solution changed from colorless to blue, and violet, which characteristic of gold nanoparticle growth.

Raman Spectroscopy and Microscopy: Two Raman confocal microscopes operating at two different laser frequencies were used. The first system is a confocal Raman microscope (CRM200, WITec, Ulm, Germany) equipped with a piezo-scanner (P-500, Physik Instrumente, Karlsruhe, Germany) and a diode-pumped 785 nm NIR laser excitation (Toptica Photonics AG, Graefelfing, Germany). The laser beam was focused through a $60 \times$ water immersion (Nikon, $\mathrm{NA}=1.0$ ) or $100 \times$ oil immersion (Nikon, $\mathrm{NA}=$ 1.25) microscope objective. The spectra were acquired with a thermoelectrically cooled CCD detector (DU401ABV, Andor, UK) behind a grating $\left(300 \mathrm{~g} \mathrm{~mm}^{-1}\right.$ ) spectrograph (Acton, Princeton Instruments Inc., Trenton, NJ, USA) with a spectral resolution of $6 \mathrm{~cm}^{-1}$.

The second system is a confocal Raman microscope (alpha300, WITec, Ulm, Germany) equipped with a frequency doubled Nd:YAG laser $(532 \mathrm{~nm}$ ) laser excitation and piezoscanner (P-500, Physik Instrumente, Karlsruhe, Germany). The spectra were acquired with a thermoelectrically cooled CCD detector (DU401A-BV, Andor, UK) placed behind the spectrometer (UHTS 300; WITec, Ulm, Germany) with a spectral resolution of $3 \mathrm{~cm}^{-1}$.

For imaging integration time of $0.1 \mathrm{~s}$ per pixel was used. The ScanCtrlSpectroscopyPlus software (version 1.38, Witec) was used for measurement and WITec Project Plus (version 2.02, Witec) for spectra processing. Calculated single spectra were exported into the OPUS software package (version 6.0) and normalized on the $\mathrm{SiO}_{2}$ $\left(485 \mathrm{~cm}^{-1}\right)$ band. A Raman imaging 3D reconstruction was obtained using Image J 1.41, Image Magic k 6.0-Q16 and Volume J 1.7a.

Cell Culture, Electropermeabilization and Co-incubation of Cells with Microparticles: NIH3T3 Fibroblasts (by ATCC) were cultured in the Dulbeco's modified eagle medium (DMEM) supplemented with $4500 \mathrm{mg}$ Glucose/L; 10\% Calf serum; 0.1\% Gentamicin (Antibiotic). Cells were seeded $6 \times 10^{3}$ cells $\mathrm{cm}^{-2}$ and incubated in an incubator with $5 \% \mathrm{CO}_{2}$ and $37^{\circ} \mathrm{C}$ for at least $4 \mathrm{~h}$ overnight before Raman measurements.

For every electropermeabilization process $1.95 \times 10^{5}$ cells were used and electropermeabilization was carried out in $200 \mu \mathrm{L}$ cytomix $\left(1.3 \times 10^{5}\right.$ cells $)$ at 500 volts for $500 \mathrm{~ms}$, repeated twice in a gold-coated electropermeabilization cuvette having an electrode gap of $2 \mathrm{~mm}$ (Bridge Bioscience Corporation) using a BioRad electroporator.

Fixation and Cytoskeleton Staining of the Tissue: for Raman imaging the cell fixation procedure was done as follows: the cell culture was removed from the scaffold dish followed with washing in phosphate buffered saline (PBS) five times, and then putted $4 \%$ of the parapharmaldehyde (PFA) and left for 15 min at room temperature, after PFA was removed and washed in PBS again five times and kept fixed in PBS for following manipulation at $4{ }^{\circ} \mathrm{C}$. In order to obtain reproducibility of Raman cell imaging, we measured over 10 single cells under the same laser and environmental conditions.

Actin cytoskeleton staining of cells in scaffold was made with the following protocol: fixation of the cell in scaffold in 4\% PFA for $5 \mathrm{~min}$; permeabilize with $0.5 \%$ triton $\mathrm{X}-100$ for $30 \mathrm{~min}$ at room temperature; washing five times in PBS; stain the scaffolds with TRITC-phalloidin 1:500 dilution for 90 min in the dark at $4^{\circ} \mathrm{C}$; wash in PBS twice briefly and then three times for 7 min each; stain the nuclei with TO-PRO-3 iodide 1:300 dilution for $5 \mathrm{~min}$ in dark at room temperature; wash the samples five times in PBS; cover samples with Vectashield mounting medium and observe under confocal microscope.

Colloidal Probe Location Inside Cells: In previous study, it was reported that microcapsules internalized through electroporation induced method are located in the cytosol of cells. ${ }^{[33]}$ In current study, colloidal probe incorporated inside cells are located in the area between the nucleus and the membrane. Signals from cellular compartments can be detected by the Raman signal.

Estimation of Signal-to-Noise Ratios: The SNR was estimated according to the following equation:

$S N R=\frac{S^{\max }-S^{b g}}{S D^{n}}$

where $S^{\max }$ is the maximum of the Raman signal at the defined peak position; $S^{\text {bg }}$ is the intensity of the background free of any Raman signal, and $S D^{n}$ corresponds to the standard deviation of the noise (obtained in the part of the spectrum free of Raman sig. nals by calculating $1 / 5$ of the peak-to-peak variation between the highest and the lowest readings).

\section{Supporting Information}

Supporting Information is available from the Wiley Online Library or from the author.

\section{Acknowledgements}

The authors thank to Susann Weichold for ESEM measurements, Christine Pilz for cell culture technical assistance and Michael Kerschnitzki for Drishti technical assistance. D.G. acknowledges support by the RFBR project (11-08-12058-ofi-m-2011). A.M., A.Y. and P.F. are grateful for support by the Alexander von Humboldt Foundation and the Max Planck Society in the framework of the Max Planck Research Award funded by the Federal Ministry of Education and Research. N.A.K. and B.S.S. are grateful for support for NSF grant \# CBET 1036672.

[1] M. Knoblauch, J. M. Hibberd, J. C. Gray, A. J. van Bel, Nat. Biotechnology 1999, 17, 906-909.

[2] R. Singhal, Z. Orynbayeva, R. V. K. Sundaram, J. J. Niu, S. Bhattacharyya, E. A. Vitol, M. G. Schrlau, E. S. Papazoglou, G. Friedman, Y. Gogotsi, Nat. Nanotechnol. 2011, 6, 57-64. 
[3] J. Lackowitz, Principles of Fluorescnce Spectroscopy. Springer Science + Business Media, New York 2006.

[4] C. Matthaus, T. Chernenko, J. A. Newmark, C. M. Warner, M. Diem, Biophys. J. 2007, 93, 668-673.

[5] A. Zoladek, F. Pascut, P. Patel, I. Notingher, Spectroscopy 2010, 24, 131-136.

[6] M. Moskovits, Rev. Modern Phys. 1985, 57, 783-826.

[7] E. C. Le Ru, P. G. Etchegoin, M. Meyer, J. Chem. Phys. 2006, 125, 204701.

[8] J. Kneipp, H. Kneipp, K. Kneipp, Chem. Soc. Rev. 2008, 37, 1052-1060.

[9] Y. Chanda Ranjit, O. Lyandres, N. C. Shah, J. A. Dieringer, R. P. Van Duyne, in Surface-Enhanced Raman Scattering - Physics and Applications (Eds: K. Kneipp, M. M. H. Kneipp) SpringerVerlag, Berlin-Heidelberg, 2006; Vol. 103, pp.367-379.

[10] S. M. Nie, S. R. Emery, Science 1997, 275, 1102-1106.

[11] D.-K. Lim, K.-S. Jeon, H. M. Kim, J.-M. Nam, Y. D. Suh, Nat. Mater. 2010, 9, 60-67.

[12] D.-K. Lim, K.-S. Jeon, J.-H. Hwang, H. Kim, S. Kwon, Y. D. Suh, J.-M. Nam, Nat. Nanotechnol. 2011, 6, 452-460.

[13] M. F. Cardinal, B. Rodriguez-Gonzalez, R. A. Alvarez-Puebla, J. Perez-Juste, L. M. Liz-Marzan, J. Phys. Chem. C 2010, 114, 10417-10423.

[14] L. D. Qin, S. L. Zou, C. Xue, A. Atkinson, G. C. Schatz, C. A. Mirkin, Proc. Natl. Acad. Sci. USA 2006, 103, 13300-13303.

[15] R. Alvarez-Puebla, L. M. Liz-Marzan, F. J. G. de Abajo, J. Phys. Chem. Lett. 2010, 1, 2428-2434.

[16] S. J. Lee, A. R. Morrill, M. Moskovits, J. Am. Chem. Soc. 2006, 128, $2200-2201$

[17] Z. N. Zhu, H. F. Meng, W. J. Liu, X. F. Liu, J. X. Gong, X. H. Qiu, L. Jiang, D. Wang, Z. Y. Tang, Angew. Chem. Int. Ed. 2011, 50, 1593-1596.

[18] C. E. Talley, J. B. Jackson, C. Oubre, N. K. Grady, C. W. Hollars, S. M. Lane, T. R. Huser, P. Nordlander, N. J. Halas, Nano Lett. 2005, 5, 1569-1574.

[19] L. Piao, S. Park, H. B. Lee, K. Kim, J. Kim, T. D. Chung, Analyt. Chem. 2010, 82, 447-451.

[20] S. Pal, L. E. Depero, I. Alessandri, Nanotechnology 2010, 21, 425701.

[21] A. Pallaoro, G. B. Braun, N. O. Reich, M. Moskovits, Small 2010, 6, 618-622.

[22] R. H. Baughman, A. A. Zakhidov, W. A. de Heer, Science 2002, 297, 787-792.

[23] T. Assmus, K. Balasubramanian, M. Burghard, K. Kern, M. Scolari, N. Fu, A. Myalitsin, A. Mews, Appl. Phys. Lett. 2007, 90, 173109.

[24] M. Scolari, A. Mews, N. Fu, A. Myalitsin, T. Assmus, K. Balasubramanian, M. Burghard, K. Kern, J. Phys. Chem. C 2008, 112, 391-396.

[25] R. A. Alvarez-Puebla, A. Agarwal, P. M. Bisnu, P. Khanal, P. Aldeanueva-Potel, E. Carbó-Argibay, N. Pazos-Pérez, E. R. Zubarev, N. A. Kotov, L. M. Liz-Marzán, Proc. Natl. Acad. Sci. 2011, 108, 8157-8161.

[26] L. Xu, H. Kuang, C. Xu, W. Ma, L. Wang, N. A. Kotov, J. Am. Chem. Soc. 2012, 134, 1699-1709.

[27] M. Dierendonck, S. De KokerR. De Rycke, P. Bogaert, J. Grooten, C. Vervaet, J. P. RemonB. G. De Geest, ACS Nano 2011, 5, 6886-6893.
[28] A. M. Pavlov, A. V. Sapelkin, X. Huang, K. M. Y. P’ng, A. J. Bushby, G. B. Sukhorukov, A. G. Skirtach, Macromol. Biosci. 2011, 11, 848-854.

[29] Y. Yan, C. J. Ochs, G. K. Such, J. K. Heath, E. C. Nice, F. Caruso, Adv. Mater. 2010, 22, 5398-5403.

[30] W. J. Zhang, K. Gilstrap, L. Y. Wu, K. C. R. Bahadur, M. A. Moss, Q. A. Wang, X. B. Lu, X. M. He, ACS Nano 2010, 4, 6747-6759.

[31] A. M. Javier, P. del Pino, M. F. Bedard, D. Ho, A. G. Skirtach, G. B. Sukhorukov, C. Plank, W. J. Parak, Langmuir 2008, 24, 12517-12520.

[32] A. G. Skirtach, A. M. Yashchenok, H. Mohwald, Chem. Commun. 2011, 47, 12736-12746.

[33] R. Palankar, A. G. Skirtach, O. Kreft, M. Bedard, M. Garstka, K. Gould, H. Mohwald, G. B. Sukhorukov, M. Winterhalter, S. Springer, Small 2009, 5, 2168-2176.

[34] B. V. Parakhonskiy, M. F. Bedard, T. V. Bukreeva, G. B. Sukhorukov, H. Mohwald, A. G. Skirtach, J. Phys. Chem. C 2010, 114, 1996-2002.

[35] A. M. Yashchenok, D. N. Bratashov, D. A. Gorin, M. V. Lomova, A. M. Pavlov, A. V. Sapelkin, B. S. Shim, G. B. Khomutov, N. A. Kotov, G. B. Sukhorukov, H. Mohwald, A. G. Skirtach, Adv. Funct. Mater. 2010, 20, 3136-3142.

[36] M. Hedegaard, C. Matthäus, S. Hassing, C. Krafft, M. Diem, J. Popp, Theor. Chem. Acc. 2011, 130, 12491260.

[37] Z. A. Combs, S. H. Chang, T. Clark, S. Singamaneni, K. D. Anderson, V. V. Tsukruk, Langmuir 2011, 27, 3198-3205.

[38] A. G. Skirtach, A. M. Javier, O. Kreft, K. Kohler, A. P. Alberola, H. Möhwald, W. J. Parak, G. B. Sukhorukov, Angew. Chem.-Int. Ed. 2006, 45, 4612-4617.

[39] M. A. Ochsenkuhn, P. R. T. Jess, H. Stoquert, K. Dholakia, C. J. Campbell, ACS Nano 2009, 3, 3613-3621.

[40] Z. Movasaghi, S. Rehman, I. U. Rehman, Appl. Spectrosc. Rev. 2007, 42, 493-541.

[41] A. M. Javier, O. Kreft, M. Semmling, S. Kempter, A. G. Skirtach, O. T. Bruns, P. del Pino, M. F. Bedard, J. Raedler, J. Kaes, C. Plank, G. B. Sukhorukov, W. J. Parak, Adv. Mater. 2008, 20, 42814287.

[42] W. Jiang, B. Y. S. Kim, J. T. Rutka, W. C. W. Chan, Nat. Nanotechnol. 2008, 3, 145-150.

[43] A. Haase, H. F. Arlinghaus, J. Tentschert, H. Jungnickel, P. Graf, A. Mantion, F. Draude, S. Galla, J. Plendl, M. E. Goetz, A. Masic, W. Meier, A. F. Thuenemann, A. Taubert, A. Luch, ACS Nano 2011, 5, 3059-3068.

[44] B.-H. Jun, D. W. Hwang, H. S. Jung, J. Jang, H. Kim, H. Kang, T. Kang, S. Kyeong, H. Lee, D. H. Jeong, K. W. Kang, H. Youn, D. S. Lee, Y.-S. Lee, Adv. Funct. Mater. 2012, 22, 18431849.

[45] R. A. Jalil, Y. Zhang, Biomaterials 2008, 29, 4122-4128.

[46] M. Brust, D. Bethell, D. J. Schiffrin, C. J. Kelly, Adv. Mater. 1995, 7, 795-797.

[47] T. Pham, J. B. Jackson, N. J. Halas, T. R. Lee, Langmuir 2002, 18, 4915-4920.

Received: June 29, 2012

Revised: August 7, 2012

Published online: October 9, 2012 